\title{
Submitted: Then Sonography of tendon pathology in the hand and wrist
}

Accepted: 15.05.2021

Published: 29.11.2021

\footnotetext{
Keywords tendon injury, hand, pulley injury, tenosynovitis, ultrasound
}

\section{Introduction}

At the center of our daily life activities, the hand is frequently exposed to both direct and indirect trauma and overuse injuries ${ }^{(1)}$. Therefore, the detection and detailed evaluation of hand injuries are common referral indications for ultrasound (US) examinations. Most injuries to the hand are open injuries. These injuries occur more commonly to the extensor tendons rather than the flexor tendons ${ }^{(2)}$. This review covers the most common injury patterns of pulleys, flexor and extensor tendons in the wrist and lesser fingers for a reader with a comprehensive knowledge of normal hand and wrist anatomy (for a detailed review of normal anatomy, please read the article by De Maeseneer et al. ${ }^{(3)}$ in a previous issue of this journal). Tendons at the wrist level can be examined with a linear US transducer $(10-18 \mathrm{MHz})$. However, abnormalities of the extensor tendons in the fingers are better assessed using a high-frequency US probe (14-33 MHz) such as a "hockey-stick" transducer with a smaller field-of-view, and a diligent scanning procedure. 


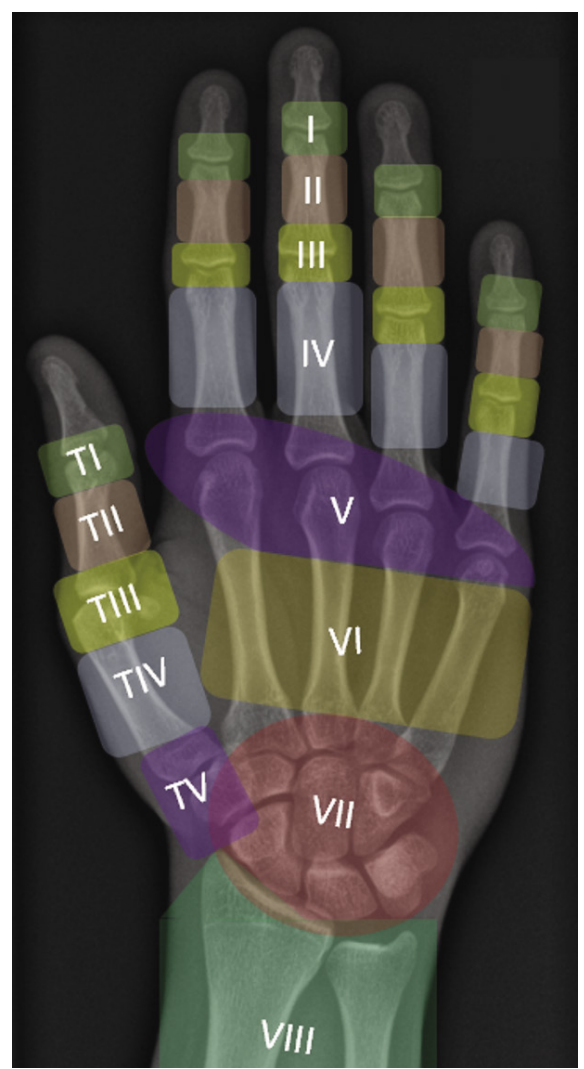

Fig. 1. Zonal classification of extensor tendon injuries: from distal to proximal, odd numbers conventionally refer to specific joint levels. In the fingers: zone I indicates the DIP joint level, zone III the PIP joint, zone V the MCP joint; in the thumb: the IP joint level is zone I, the MCP joint is zone III

\section{Extensor tendons}

\section{Tears and ruptures}

Extensor tendon tears may be the result of open injuries or closed ruptures. The classification of extensor tendon injuries into anatomical zones (by Kleinert and Verdan, Fig. 1) and the evaluation of injury characteristics by imaging are essential tools for the selection of the appropriate treatment ${ }^{(4)}$. In every case of a tendon tear, the radiologist should report the following details for the referring surgeon ${ }^{(5)}$ :

- exact location of the tear;

- tear type: complete or partial-thickness;

- in partial-thickness tears: the percentage of the tendon involved;

- in cases of a complete tendon tear: the degree of tendon retraction and associated avulsion injury, including the degree of displacement of the avulsed bone fragment.

Full-thickness tears present on US as an absolute disruption of the tendon continuity (Fig. 2) ${ }^{(6,7)}$. In acute indirect injury, tendon stumps are typically thickened and appear hypoechoic with inhomogeneities and loss of the normal fibrillar echotexture. After direct injury, the stumps typically appear even. Both static and dynamic US scanning should be used to assess the gap between the tendon stumps in order to inform the surgeon`s incision planning.

Partial-thickness tears typically present on US as an incomplete interruption in tendon continuity or focal
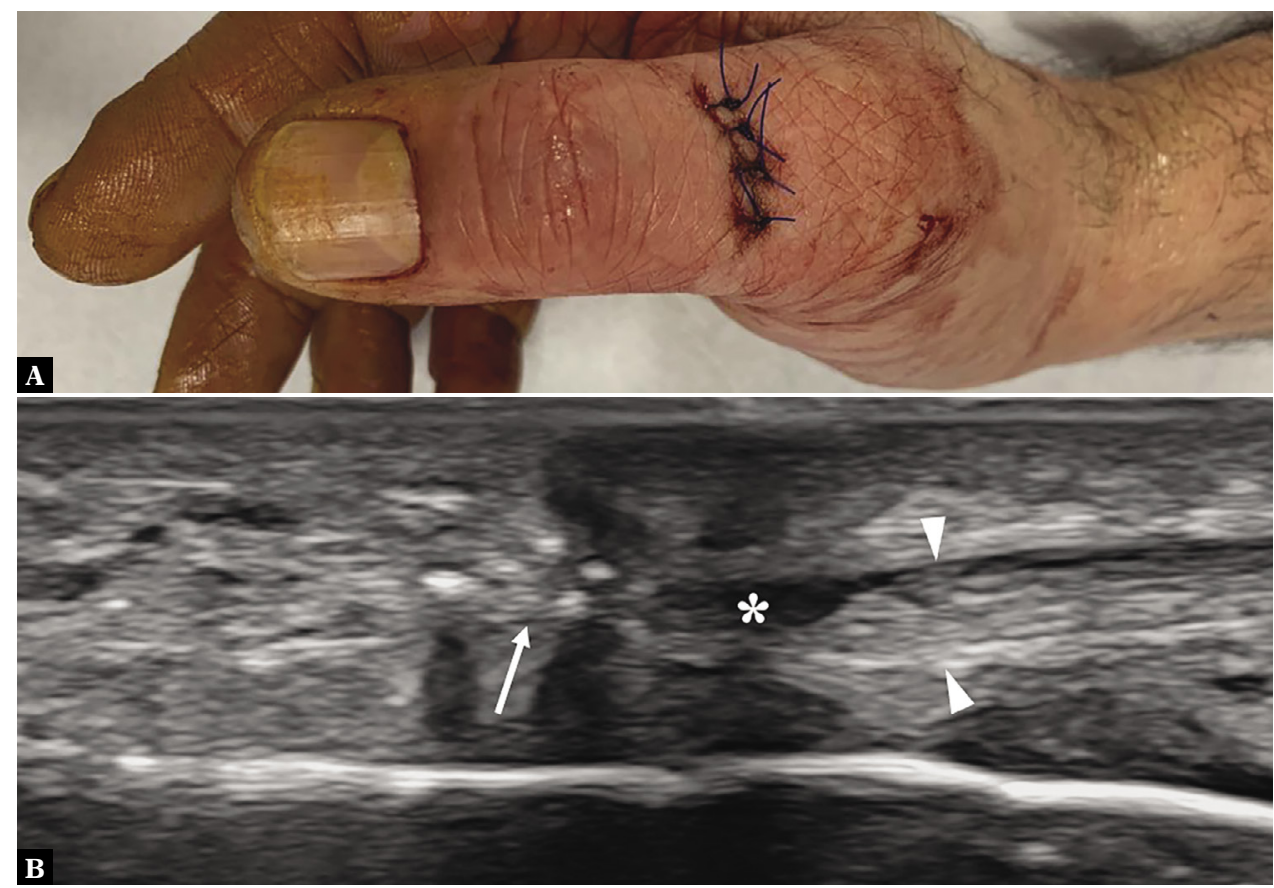

Fig. 2. Extensor pollicis longus full-thickness tear. A. Photograph showing a sutured wound over the dorsal thumb due to a penetrating injury by a glass fragment. B. After repair, long-axis 22-8 MHz. US image shows signs of extensor pollicis longus retear. Note the subtotal discontinuity of the proximal tendon end (arrowheads) which appears retracted away from the sutures (thin arrow). Mild fluid (asterisk) fills the gap 


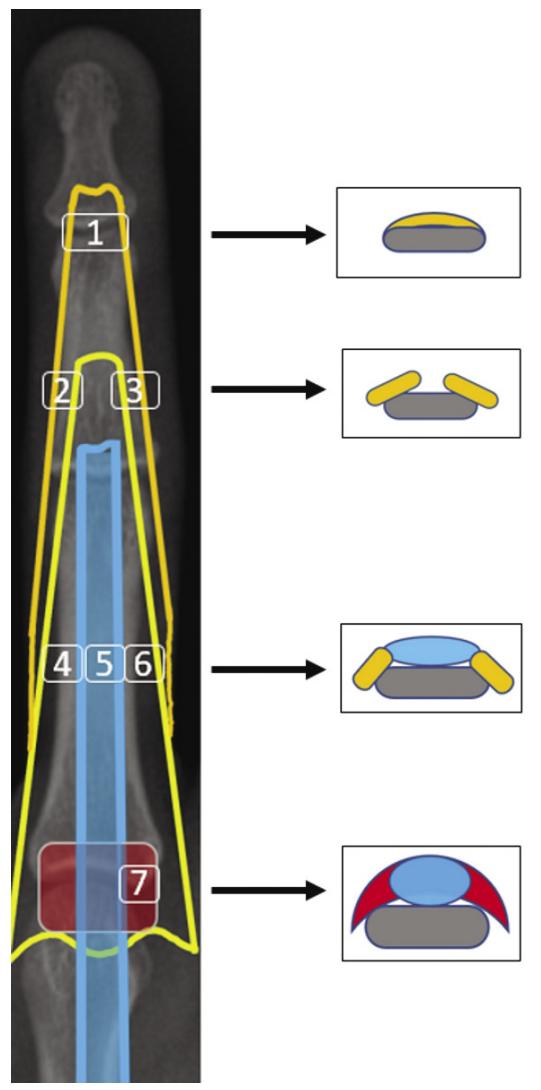

Fig. 3. Schematic drawing of the extensor mechanism of the fingers with corresponding transverse slices: (1) terminal tendon, (2) lateral conjoined tendon, (3) medial conjoined tendon, (4) lateral slip, (5) central slip, (6) medial slip (7) sagittal bands

disturbance of the parallel fiber lining. A hypoechoic or anechoic tendon defect is commonly observed, along with fusiform tendon swelling ${ }^{(6,7)}$. Furthermore, a hematoma in the synovial tendon sheath is often seen in acute settings. In chronic lesions, hypoechoic areas with fibrosis and adhesions typically surround the ruptured tendon. Bony fragments in cases with associated fractures may present as hyperechoic areas with posterior shadowing on US imaging ${ }^{(5,7)}$. An US finding of an uninterrupted, hypomobile tendon is often reported in healed finger injuries due to tendon elongation. In such cases, the examiner can use passive flexion and extension movements of the finger (and its comparison to other fingers or the contralateral side) in order to detect any mild gliding abnormalities.

\section{Mallet finger}

The term "mallet finger" or "baseball finger" refers to a deformity of the finger due to injury of the extensor tendon at its insertion point along the base of the distal phalanx $x^{(5,7,8)}$ (zone \#1 injury; Fig. 3 and Fig. 4). The terminal portion of the tendon may rupture directly, or it may be torn from the insertion point with a small piece of bone, i.e., a so-called "bony avulsion injury" (Fig. 4). The typical injury mechanism is a sudden, forceful hyperflexion of an

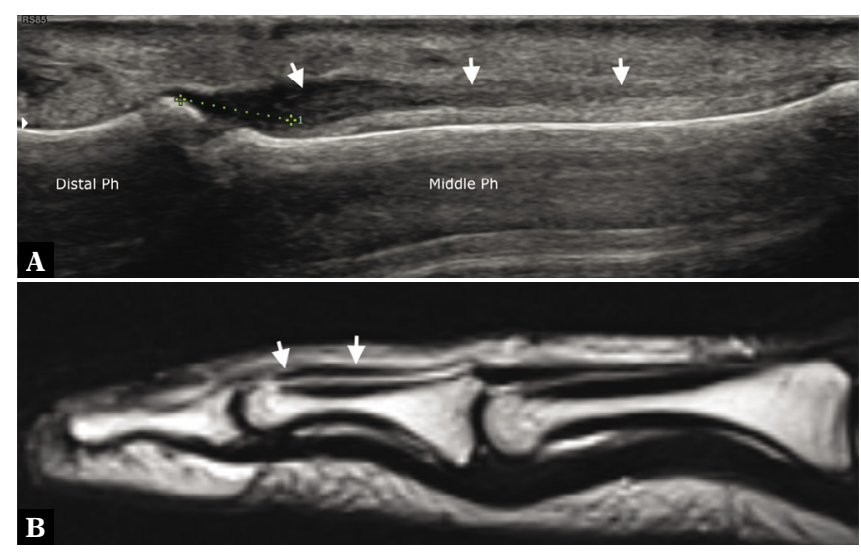

Fig. 4. Mallet finger. A. On a longitudinal sonogram, the extensor tendon of the fourth finger (arrows) is completely torn from the distal phalanx (Distal Ph) base. The tendon stump is thickened and hypoechoic at the level of the distal third of the middle phalanx (Middle Ph). Tendon retraction (caliper) measured 4-5 $\mathrm{mm}$. B. On a longitudinal T2-weighted MRI image, the extensor tendon retraction (arrows) is shown in the same patient

extended DIP joint, such as when a flying ball strikes the fingertip ${ }^{(8)}$. If left untreated, a mallet finger may progress to a DIP joint flexion deformity and eventually result in a swan neck deformity.

Typical US findings in mallet fingers include a defect at the insertion point observed as a space during DIP flexion that closes during DIP extension, a swollen tendon over the distal middle phalanx, and a hypomobile deep flexor tendon ${ }^{(9)}$.

If bone is not involved, mallet finger injuries are stabilized in a splint for several weeks. Surgery is recommended for cases with complex injury patterns, such as fractures involving more than $30-50 \%$ of the joint surface or posttraumatic malalignment of the DIP joint, in order to prevent development of a swan neck deformity or a prolonged extension deficit ${ }^{(8)}$.

\section{Central slip lesion}

Less common sports injuries are closed disruptions of the central slip (Fig. 3) at the base of the middle phalanx. They may occur with or without bony fragment avulsion ${ }^{(5,10,11)}$ (zone \#3 injury, Fig. 1 and Fig. 5). In the acute phase, detection of central slip lesions may be difficult by clinical examination alone. The PIP joint may still extend due to the intact lateral slips. If a central slip lesion is overlooked, the patient often returns after 2-3 weeks with a so-called "boutonnière deformity" (Fig. 5). This term refers to a flexion deformity of the PIP joint and hyperextension of the DIP joint ${ }^{(12)}$ and is caused by an increased triangular ligament tension resulting in palmar migration of the lateral slip. The boutonnière deformity often remains permanent since the tendon will heal with splinting, but in a lengthened position. Therefore, the key to a good outcome after central slip rupture is an early diagnosis and initiation of a full-time splinting ${ }^{(12)}$. 


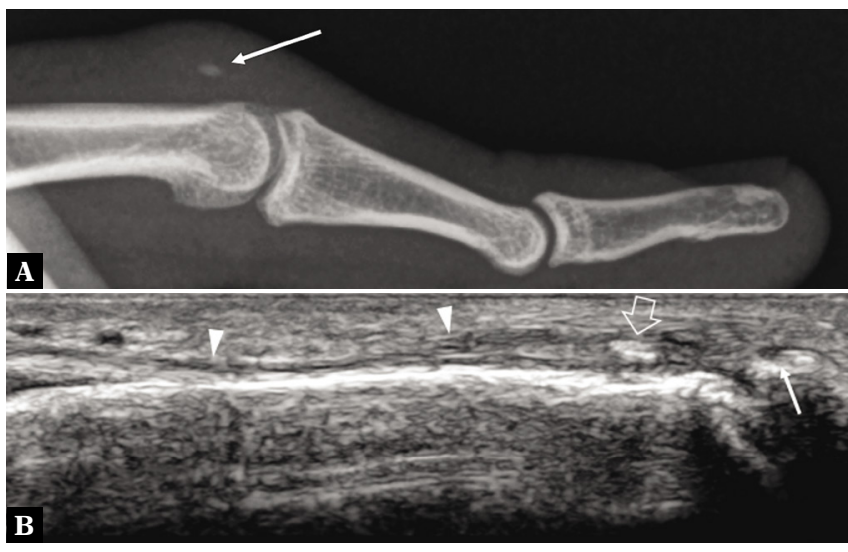

Fig. 5. Boutonniere deformity. A. Lateral radiograph and B. corresponding long-axis 17-5 MHz US image of the middle finger demonstrate a small piece of bone (arrow) avulsed from the base of the middle phalanx. In $\mathbf{B}$, the donor site of avulsion (thin arrow) is shown as a small concavity at the base of the middle phalanx. The retracted fragment (large arrow) is in continuity with the central slip (arrowheads) of the extensor hood

For diagnosis, the US probe should be placed over the dorsal PIP joint in longitudinal alignment with the central slip. A pre-insertional extensor mechanism defect and impaired central slip gliding in finger flexion and extension are the primary diagnostic factors. Unfortunately, the anomalous location of the displaced lateral slip is more difficult to detect with US.

\section{Sagittal band disruption}

Sagittal bands are the most important component of the dorsal extensor hood at the metacarpophalangeal (MCP) joint (Fig. 3). The superficial and deep layers of the sagittal bands attach to the palmar plate volarly and to the extensor tendon dorsally ${ }^{(3)}$. The extensor digitorum communis (EDC) tendon is stabilized at the MCP joint and protected from subluxation during digital movement by the sagittal bands. The radial band is thinner and weaker than the ulnar band. Closed disruption of an EDC tendon sagittal band (zone \#5 injury; Fig. 6) is a common injury after direct trauma to the proximal phalanx or the dorsal aspect of the MCP joint. Most often the third digit ${ }^{(10,13)}$ is affected.

Injuries to the sagittal band are classified into three types ${ }^{(5)}$ :

- local contusion (type-1);

- subluxation (type-2);

- dislocation (type-3) of the EDC tendon to the volar aspect of the MCP joint.

Consequently, the EDC tendon may dislocate to the radial or ulnar side, such that the finger cannot actively extend at the MCP joint ${ }^{(14)}$. Radial-sided ruptures of the sagittal band in particular frequently result in tendon instability.

In dynamic US, sporadic snapping of the EDC tendon to one side (subluxation) or dislocation of the tendon between the metacarpal heads can be observed when the fist is clenched $^{(13)}$.

Acute injuries are treated with immobilization (splint in joint extension), whereas chronic lesions require surgical reconstruction of the injured sagittal band.

\section{Extensor tenosynovitis}

The term "tenosynovitis" is defined as any inflammation and swelling of the synovial sheath. Inflammatory processes, trauma, and overuse injuries lead to damage of the extrinsic extensor tendons and the surrounding tendon
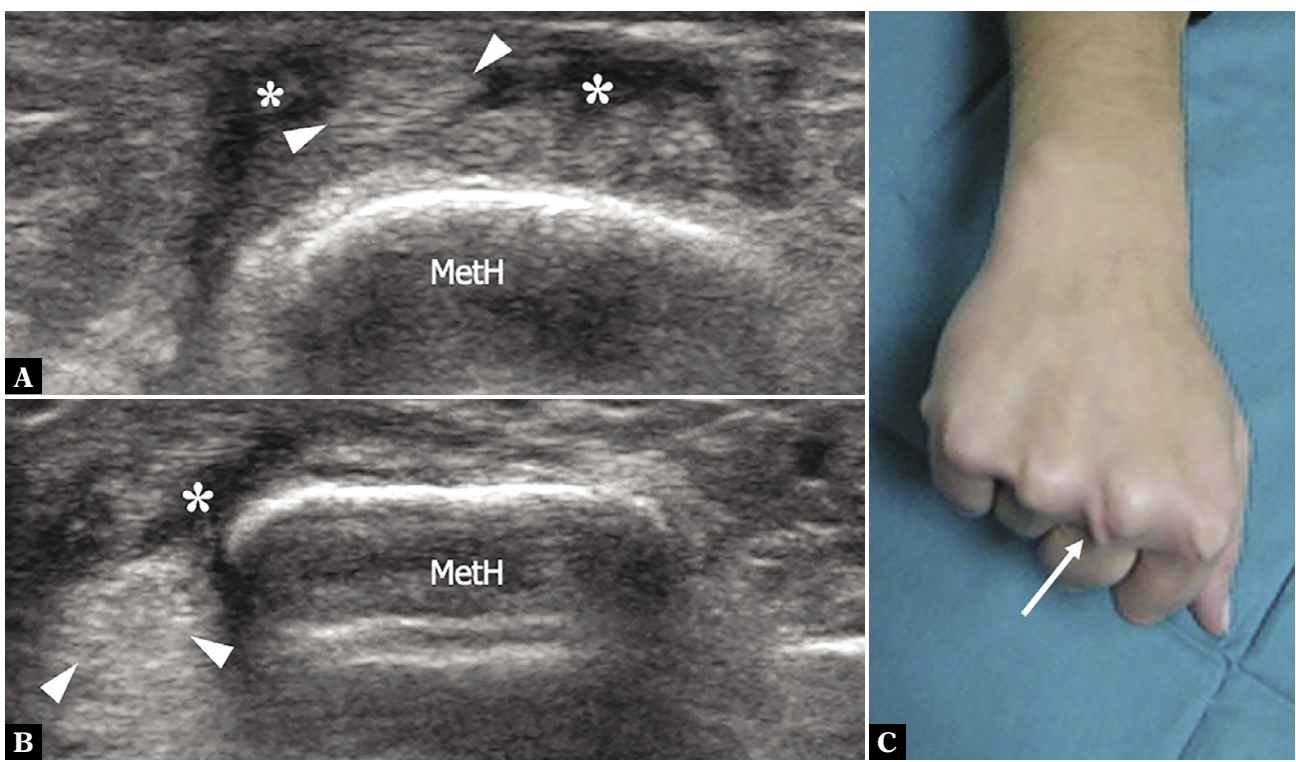

Fig. 6. Sagittal band injury. A, B. Transverse 17-5 MHz. US images over the dorsal aspect of the metacarpal head (MetH) of the right third finger acquired in extension (A) and in clenched-fist (B) position show transient ulnar dislocation of the common extensor tendon (arrowheads) during flexion. Mild local effusion (asterisks) is observed along the tendon path. C. Correlative photograph showing tendon dislocation (arrow) on the ulnar side of the third metacarpal head 


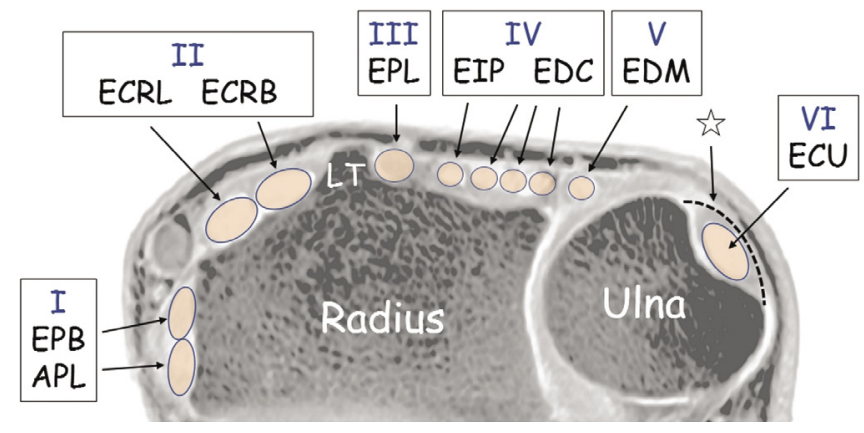

Fig. 7. Schematic drawing of the six extensor tendon compartments of the wrist, labeled from I-VI, adapted from ${ }^{(34)}$. APL - abductor pollicis longus; EPB - extensor pollicis brevis; ECRL - extensor carpi radialis longus; ECRB - extensor carpi radialis brevis; $E P L$ - extensor pollicis longus; EIP - extensor indicis proprius; $E D C$ - extensor digitorum communis; EDM - extensor digiti minimi; ECU - extensor carpi ulnaris; Asterisk - subsheath; LT - Lister's tubercle; Note the retinacula for each compartment are removed for better visibility sheaths ${ }^{(15)}$. In chronic tenosynovitis, one will find tears and ruptures of the tendons, as well as neighboring bony changes such as erosions or periosteal reactions.

Tenosynovitis may affect each extensor compartment (Fig. 7) at the level of the wrist ${ }^{(16)}$.

\section{First and second compartments}

Tenosynovitis occurs most commonly in the first compartment as “De Quervain`s syndrome” (Fig. 8). This chronic injury stems from the overuse and repetitive movement of the extensor pollicis brevis (EPB) and the abductor pollicis longus (APL) tendons under the retinaculum at the level of the distal radius ${ }^{(17)}$. Initially, this manifests as tendon edema, ensuing constriction of the tendon against the retinaculum, and thickening of the retinaculum, caused by microinjuries and impaired healing.
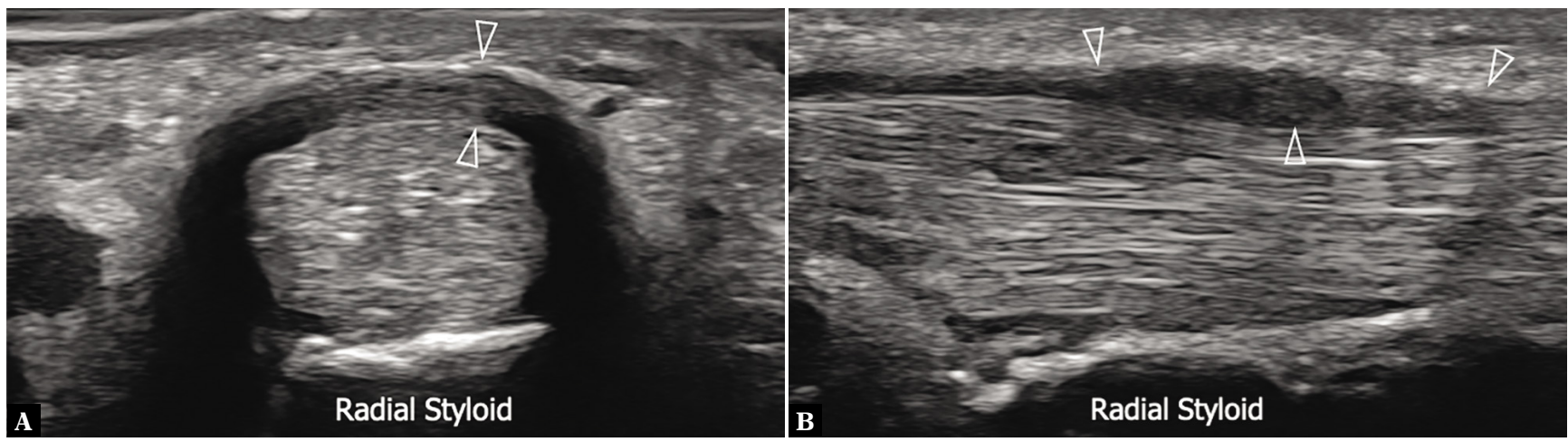

Fig. 8. De Quervain's syndrome. Short-(A) and long-axis (B) 24-8 MHz US images over the radial styloid reveal a thickened, hypoechoic retinaculum (arrowheads) and the swollen abductor longus and extensor pollicis brevis tendons. Note that the two tendons form a rounded complex and cannot be separated from each other, being constricted by the abnormal retinaculum
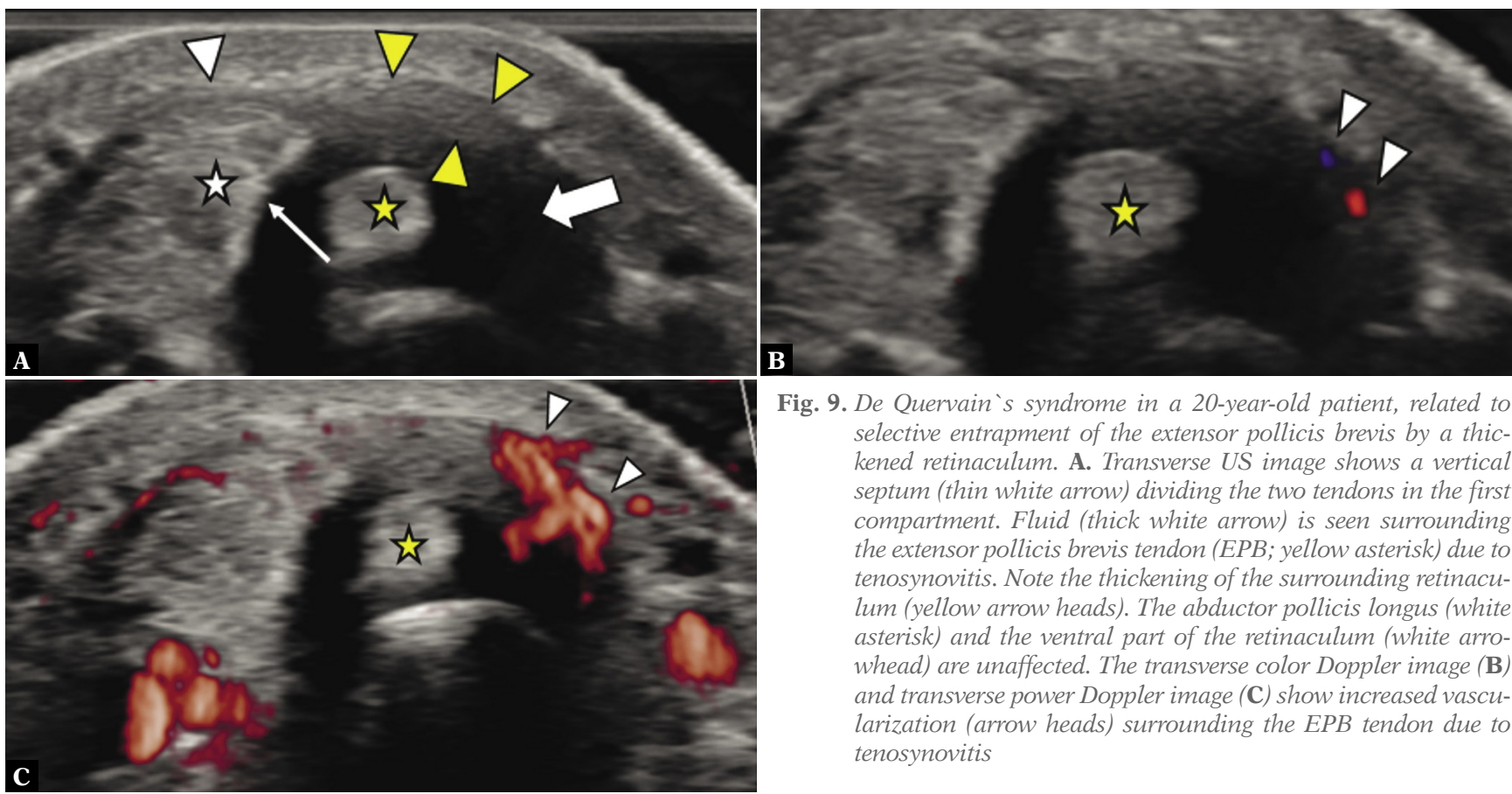

Fig. 9. De Quervain's syndrome in a 20-year-old patient, related to selective entrapment of the extensor pollicis brevis by a thickened retinaculum. A. Transverse US image shows a vertical septum (thin white arrow) dividing the two tendons in the first compartment. Fluid (thick white arrow) is seen surrounding the extensor pollicis brevis tendon (EPB; yellow asterisk) due to tenosynovitis. Note the thickening of the surrounding retinaculum (yellow arrow heads). The abductor pollicis longus (white asterisk) and the ventral part of the retinaculum (white arrowhead) are unaffected. The transverse color Doppler image (B) and transverse power Doppler image (C) show increased vascularization (arrow heads) surrounding the EPB tendon due to tenosynovitis 

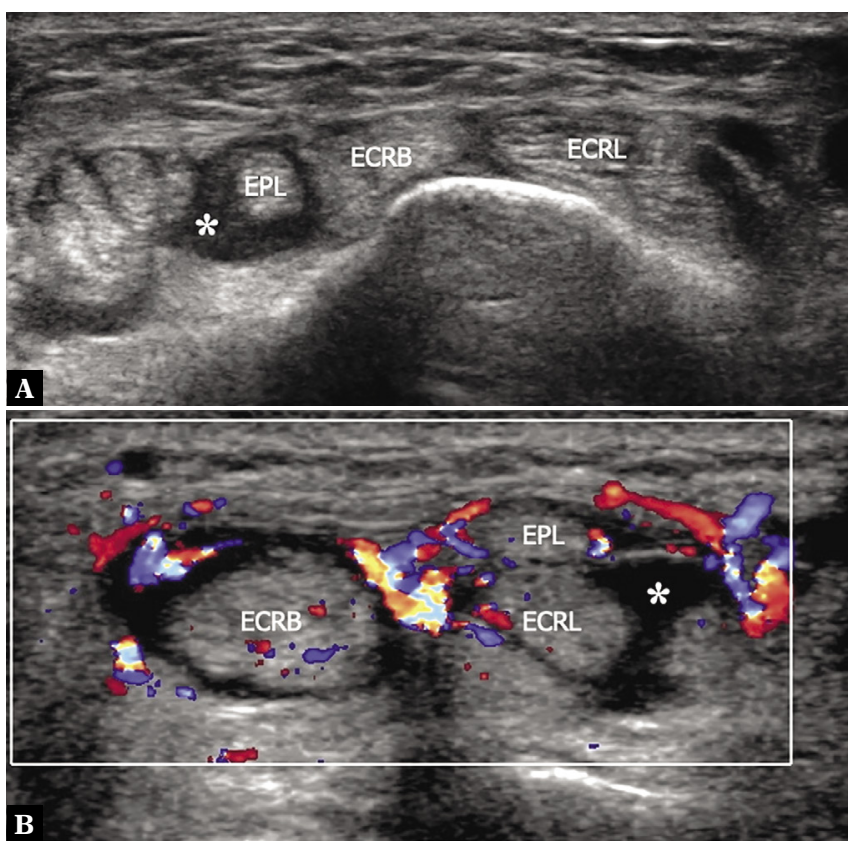

Fig. 10. Distal intersection syndrome. A. Proximal to the criss-crossing point, transverse 17-5 MHz US image shows a distended sheath (asterisk) of the extensor pollicis longus (EPL) as it runs alongside the ulnar aspect of the extensor carpi radialis brevis $(E R C B)$ and extensor carpi radialis longus (ECRL). B. More distally, transverse 17-5 MHz color Doppler US image demonstrates the extensor pollicis longus (EPL) as it crosses over the extensor carpi radialis brevis (ECRB) and longus (ECRL) tendons. The sheath of the second and third compartments appears mildly distended by tenosynovitis with effusion (asterisk). Note diffuse local hyperemia and the swollen appearance of the ECRB

Dynamic US scanning, preferably in the long-axis view, may detect real-time gliding of the obstructed tendon under the retinaculum ${ }^{(17)}$. The normal oval shape of the EPB and APL tendons on short-axis view typically changes to a rounder appearance in chronic settings. Thickened tendons also lead to partial tears of the retinaculum. In the acute stage, increased vascularization is observed in both the tendons and the retinaculum, whereas in chronic conditions, thickening and fibrotic lesions are observed. Sometimes a vertical septum is observed as having divided the first compartment into two discrete tunnels and increasing the risk of tenosynovitis. In such cases, only the EPB is typically involved, while the APL tendon remains unaffected (Fig. 9). Common causes of De Quervain`s syndrome include sporting activities that require repetitive flexion or extension of the wrist in combination with thumb abduction against resistance. This syndrome develops frequently during new motherhood ("baby wrist") due to hormonal changes and an incorrect wrist position while lifting the baby ${ }^{(18)}$.

Proximal intersection syndrome (also called crossover syndrome, peritendinitis crepitans or oarsmen's wrist) is a type of overuse injury presenting clinically with pain and swelling at the distal forearm, some centimeter proximal to Lister's tubercle. Here lies the intersection point of the first (APL and EPB) and the second extensor compartment (extensor carpi radialis longus (ECRL) and extensor carpi radialis brevis (ECRB) tendons) ${ }^{(19)}$. US may detect edematous changes in the APL and EPB (typically at the myotendinous junctions) and the loss of a hyperechoic plane deviding the two different compartments ${ }^{(5,20)}$. Other findings include sheath effusion and swelling, typically of the tendons in the second extensor compartment. Sometimes even ganglion cysts may be seen.

\section{Second and third compartments}

Distal intersection syndrome occurs at the level of Lister's tubercle, where the extensor pollicis longus (EPL) tendon, (i.e., third extensor compartment) crosses over the second extensor compartment tendons ${ }^{(16)}$. The syndrome is often
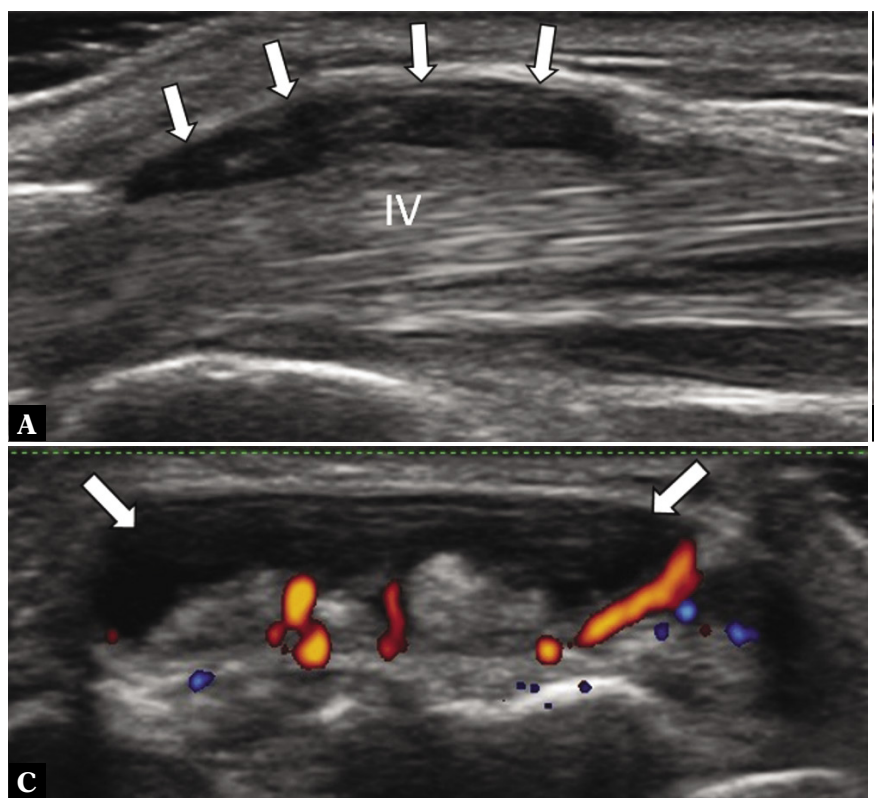

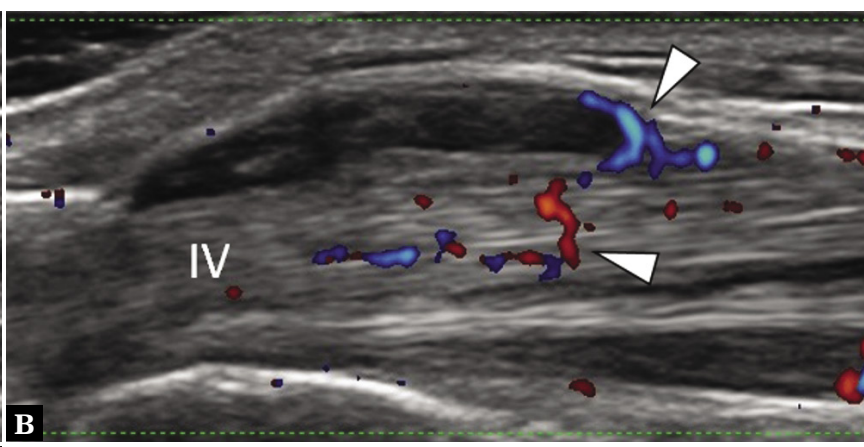

Fig. 11. Tenosynovitis of the fourth extensor compartment in a patient with psoriatic arthritis. A. Longitudinal US image with fluid, thickened synovium, and thickened retinaculum (white arrows) around the tendons in the fourth extensor compartment and increased vascularity on longitudinal (B) and transverse color Doppler (C) images, both in the synovium and the tendons 

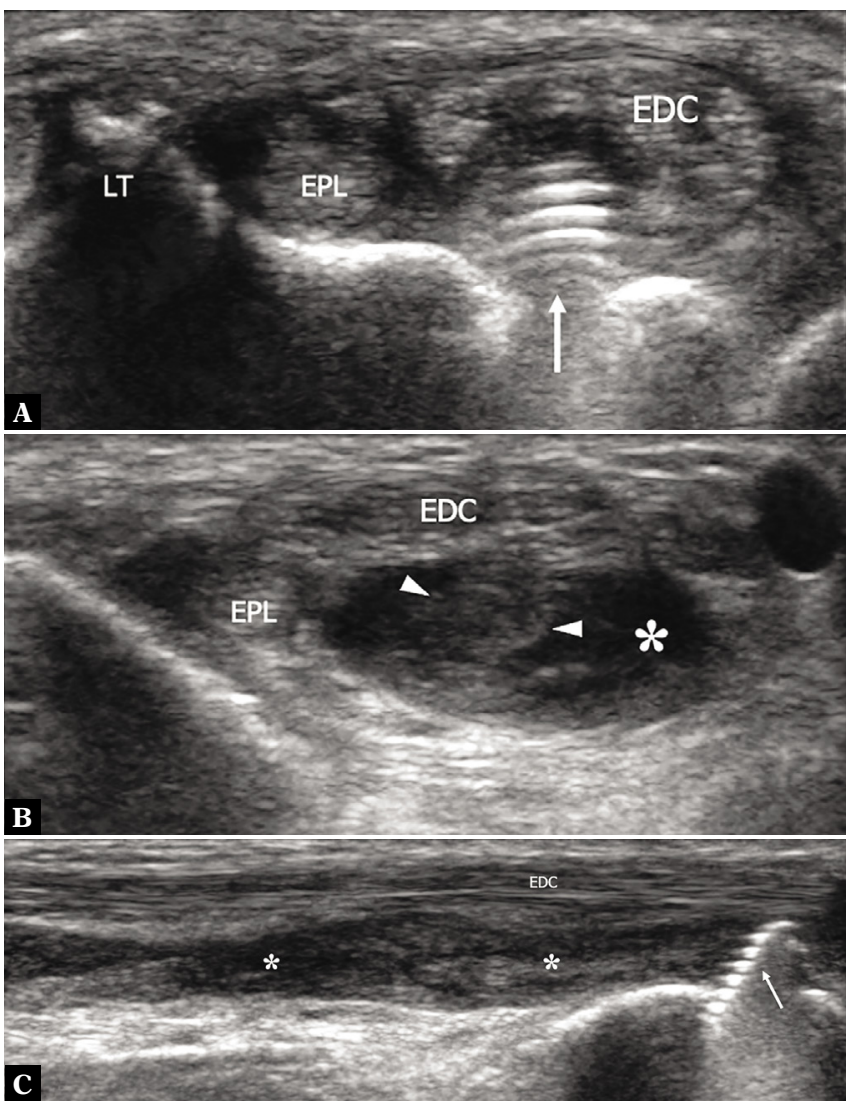

Fig. 12. Screw tip impingement and tear of the extensor indicis proprius tendon in a 45-year-old woman after volar plating for distal radial fracture. A. Transverse 17-5 MHz US image obtained at the level of Lister's tubercle (LT) reveals the thread and tip of a screw (thin arrow) impinging the extensor indicis proprius tendon. Note the intact extensor pollicis longus (EPL) and the displaced slips of the extensor digitorum communis (EDC). B. Transverse 17-5 MHz US image obtained proximal to A demonstrates the retracted extensor indicis proprius (arrowheads) surrounded by effusion and debris (asterisk). C. Longitudinal 17-5 MHz US image demonstrates the screw (thin arrow) and the empty sheath (asterisks) of the extensor indicis proprius filled with debris and hypoechoic effusion. Note the normal-appearing tendons of the extensor digitorum communis $(E D C)$ as they run more superficially

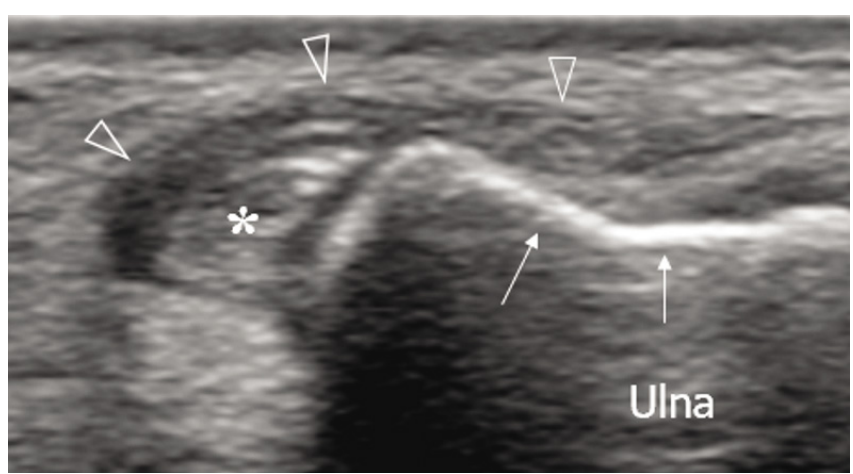

Fig. 13. Extensor carpi ulnaris (ECU) instability. ECU instability in a patient with long-standing rheumatoid arthritis. US image demonstrates the ECU tendon (white asterisk) dislocated out of the groove (white arrows). The subsheath (void arrowheads) appears lax, wavy, and displaced out of the groove caused by mechanical rubbing between the tendons due to repeated flexion and extension movements of the wrist. Sheath effusion is typically observed around the EPL tendon proximal and distal to the intersection ${ }^{(21)}$ (Fig. 10). During US examination, the carpal bones and joints should also be checked carefully along the full length of the tendon course, for impingement of tendons by underlying spurs or osteophytes. This syndrome can also be seen secondary to scaphoid fractures, or in patients with a scaphoid lunate advanced collapse (SLAC) wrist.

Non-displaced Colles fractures may cause EPL tendon rupture, whereas the stabilizing screw tips in a stabilized Colles fracture may protrude dorsally, with possible impingement of the EPL tendon ${ }^{(22)}$.

\section{Fourth compartment}

Patients with inflammatory or infectious disorders often present with EDC tenosynovitis (Fig. 11) ${ }^{(16,23)}$. US with Doppler imaging helps to assess the activity level of inflammatory diseases and to monitor treatment effects. Tendon impingement with signs of tenosynovitis may be seen in the postoperative setting, such as following treatment of distal radius fractures with screw tip impingement (Fig. 12). Furthermore, an anomalous extensor indicis proprius muscle passing with the EDC tendon in the fourth compartment may also cause tenosynovitis, known as "extensor indicis proprius syndrome"(24).

\section{Fifth compartment}

The extensor digiti minimi (EDM) tendon is known for frequent anatomic variations with a bifurcated tendon (proximal, distal, or at level of the retinaculum), often with a concomitant synovial septum. Some studies suggested that a distally bifurcated tendon might impinge on the synovial septum during full finger flexion, inducing tenosynovitis $^{(25)}$. The EDM lies close to the distal radioulnar joint (DRUJ). Therefore, EDM tenosynovitis frequently occurs in patients with arthritis of the distal radioulnar joint (DRUJ). In severe tenosynovitis, synovial pannus may infiltrate the DRUJ capsule, leading to rupture of the adjacent retinaculum, ulnar head dorsal subluxation, and tendinopathy ${ }^{(26)}$. Dynamic US in varying degrees of pronation and supination might be helpful in order to assess the tendon position in relation to the distal radioulnar joint.

\section{Sixth compartment}

A retinaculum-like structure known as the "subsheath" holds the extensor carpi ulnaris (ECU) tendon within a groove in the ulnar head ${ }^{(5,16)}$. Chronic repetitive stress may lead to stenosing tenosynovitis of the subsheath. Typical ultrasound findings include fibrosis, reactive tenosynovial effusion, and thickening of the extensor retinaculum. A weak or torn subsheath may cause ECU tendon instability or dislocation out of the ulnar groove (Fig. 13). 


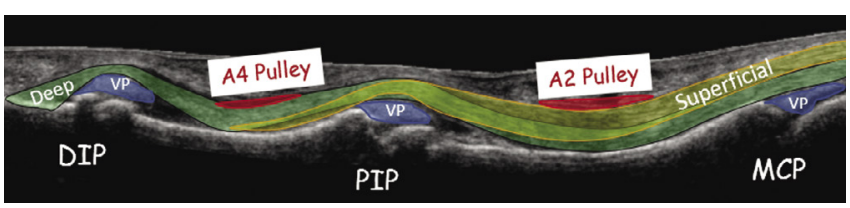

Fig. 14. Drawing of the flexor tendons of the finger, superimposed on US image. Superficial - flexor digitorum superficialis tendon (yellow), Deep - flexor digitorum profundus tendon (green), DIP - distal interphalangeal joint, PIP - proximal interphalangeal joint, $M C P$ - metacarpophalangeal joint, $V P$-volar plate

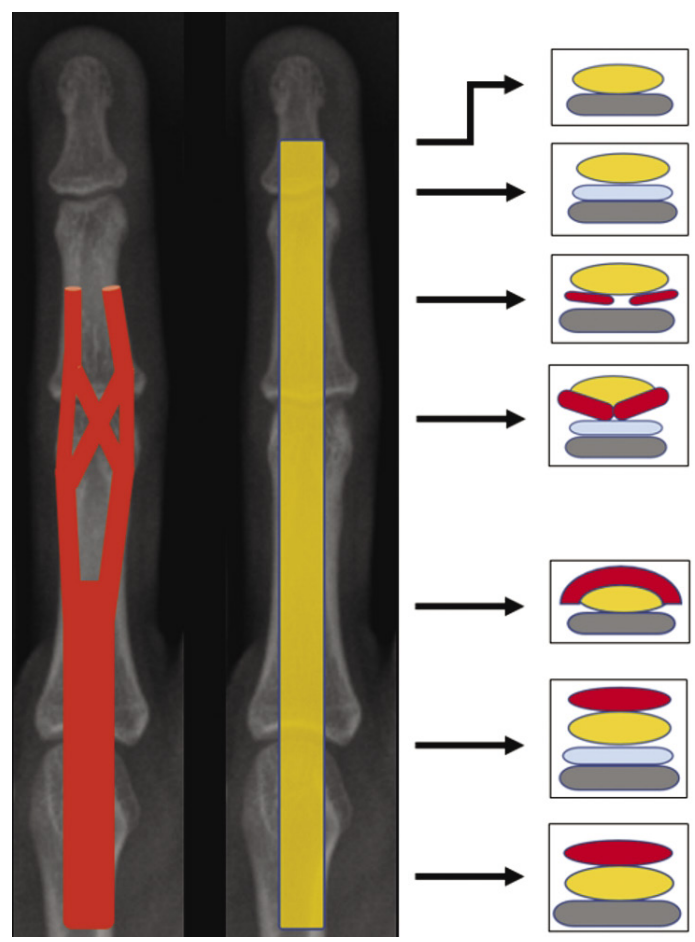

Fig. 15. Schematic drawing of superficial (red) and deep (orange) finger flexor tendons with corresponding transverse slices. Palmar plate (blue), bone (grey)

Typical causes of subsheath tears include recurrent stress injuries (e.g., in racket sports), an abrupt twisting(27), and severe DRUJ arthritis. Note: In healthy subjects the ECU tendon "dislocates" partially with supination of the forearm (up to $50 \%$ of the groove`s width) and relocates with pronation. This can be nicely seen with dynamic ultrasound.

\section{Flexor tendons and pulleys}

High resolution US provides reliable evaluation of the flexor tendons of the finger (for anatomy, see Fig. 14 and Fig. 15) and distinction of tenosynovitis, partial-thickness tendon tears, and complete tears ${ }^{(28)}$.

\section{Traumatic flexor tendon injuries}

Using the anatomical classification system developed by Kleinert and Verdan ${ }^{(4)}$, flexor tendon injuries are classified into 5 zones (Fig. 16). Flexor tendon injuries of the third,

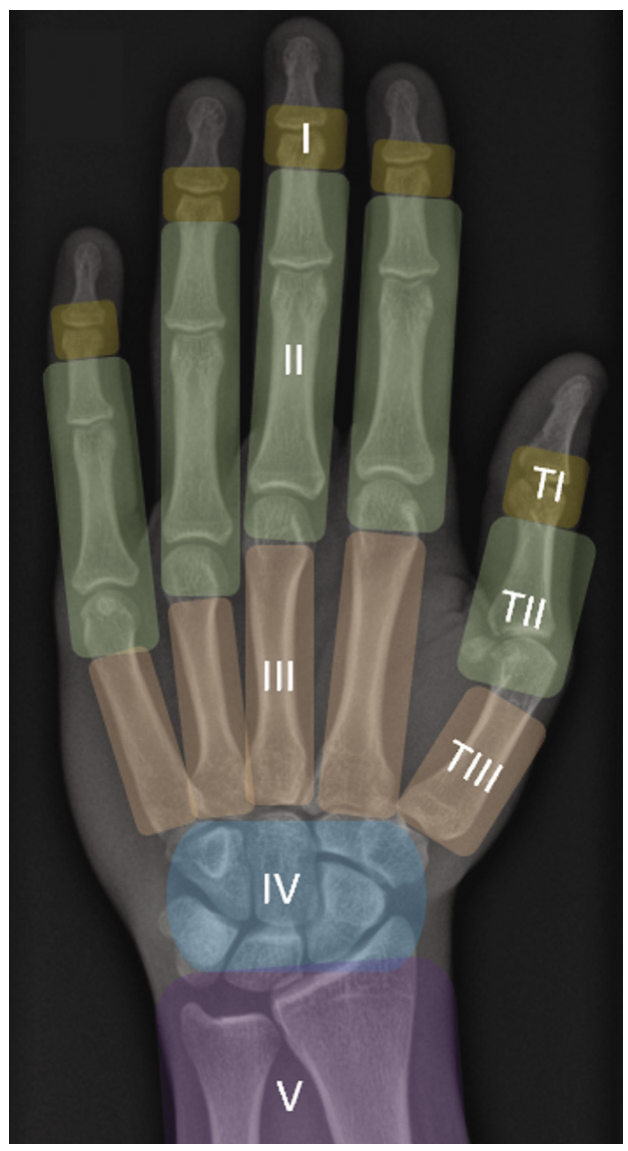

Fig. 16. Zonal classification of flexor tendon injuries: Zone I distal to the flexor digitorum superficialis (FDS) insertions, Zone II between the FDS insertions and the level of A1 pulleys, Zone III between the proximal aspect of A1 pulleys and the lumbricals origin from the flexor digitorum profundus (FDP) tendons, Zone IV at the carpal tunnel region, Zone $V$ from the distal myotendinous junction to the carpal tunnel, TI distal thumb to the IP joint, TII between thumb IP joint and A1 pulley, and TIII at the thenar eminence

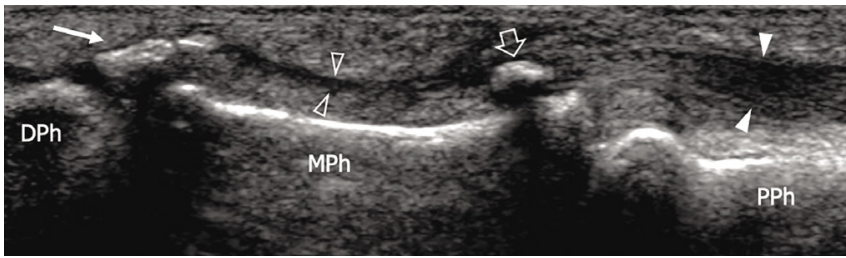

Fig. 17. Jersey finger. Long-axis 12-5 MHz US image of the middle finger reveals fracture of the volar aspect of the base of the distal phalanx with fragmentation (thin arrow) and proximal migration of a small piece of bone (void arrow) up to the distal edge of the A3-pulley. Note the flexor digitorum profundus tendon (white arrowheads) attached to the avulsed fragment and the empty sheath (void arrowheads) distal to it. DPh - distal phalanx; MPh-middle phalanx; PPh-proximal phalanx

fourth and fifth digits are frequently open, caused by laceration, and often involve the mid-substance, but not the tendon insertion point ${ }^{(11)}$. These injuries involve the flexor digitorum superficialis (FDS) tendon, the flexor digitorum profundus (FDP) tendon, or both. Injuries in Zones II to V injuries that involve the neurovascular bundle require urgent surgical 

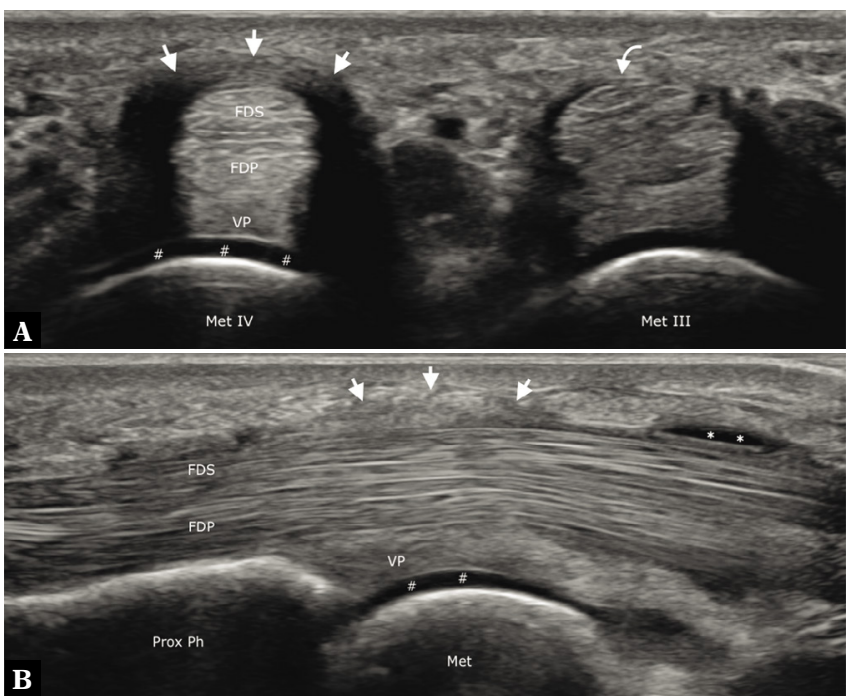

Fig. 18. Trigger finger. A. On an axial sonogram at the level of the fourth metacarpal head (Met IV), the A1-pulley (straight arrows) is thickened $(1.5 \mathrm{~mm})$. Its collateral ligaments appear artifactually hypoechoic due to anisotropy. Note the healthy A1-pulley (curved arrow) at the level of the third metacarpal head (Met III). B. On a longitudinal sonogram, A1-pulley thickening is shown. The flexor digitorum superficialis (FDS) and flexor digitorum profundus (FDP) tendons show fibrillar echotexture and synovial sheath effusion (asterisks) is seen proximally. Hash marks indicate articular cartilage; Met - metacarpal; Prox $P h$ - proximal phalanx; $V P$-volar plate

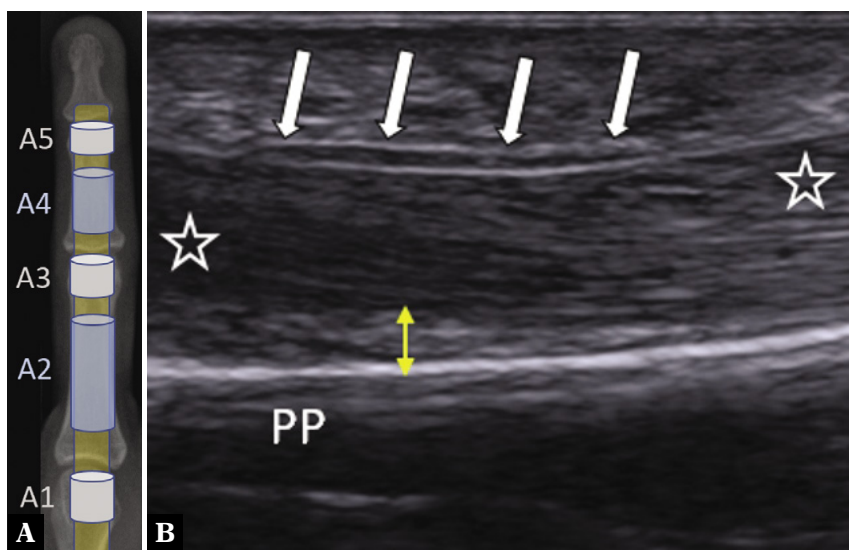

Fig. 19. Schematic drawing of annular pulleys (A) and longitudinal US image (B) at the level of the proximal phalanx (PP) with normal thin appearance of the A2-pulley ligament (white arrows). Note the normal tendon-to-bone distance (TBD) of $<2 \mathrm{~mm}$ (yellow double arrow). Flexor tendons (asterisks)

repair (i.e., within 24 hours), particularly in Zone $\mathrm{II}^{(11) \mathrm{c}}$. US permits measurement of cross-sectional flexor tendon thickness (i.e., when a tendon strain is suspected) and easy comparison with the contralateral finger ${ }^{(29)}$.

\section{Jersey finger}

The term "jersey finger" refers to a closed avulsion injury of the distal FDP tendon ${ }^{(27)}$ (Fig. 17) that occurs when a DIP joint in active flexion is forcefully hyperextended.
For example, in rugby or football games, a player snatches another player's jersey with the fingertips as that player moves away ${ }^{(11)}$. The fourth digit is frequently affected. FDP tendon avulsion injuries are assessed based on the extended Leddy and Packer classification ${ }^{(30)}$ and largely require operative treatment. Type I injuries (i.e., FDP tendon retraction to the palm) often have compromised vascular flow and require urgent operative treatment within 7 to 10 days. Treatment options for chronic avulsion injuries older than 6 weeks depend on the function level of the $\operatorname{digit}^{(30)}$.

\section{Tenosynovitis of flexor tendons}

Common causes of flexor tenosynovitis include overuse, local trauma, inflammatory arthritis, and infection ${ }^{(31)}$.

Acute flexor tenosynovitis is characterized on US by hypoechoic or anechoic effusion in the synovial sheath, increased vascularization and distention of the tenosynovium. Furthermore, the echotexture in the involved tendon lacks the typical fibrillar pattern and the tendon appears thickened. Peritendinous edema is evident in some cases $^{(28)}$. Increased vascularization due to neo-vessels can be visualized with US color or power doppler in the tendon and the surrounding soft tissues ${ }^{(28)}$.

In subacute and chronic cases of flexor tenosynovitis, the synovial sheath is thickened on US, and the flexor tendon is blurred ${ }^{(16)}$. In infectious tenosynovitis and wound injuries, foreign bodies may be observed. In comparison, rice bodies may be found within the synovial sheath effusion in inflammatory arthritis, visualized as numerous, well-defined, floating echoic masses. US may be used for guided treatment with steroid injections, or for assessment of therapeutic treatments, with a successful response demonstrating decreased inflammatory activity.

In the fingers, the flexor tendons run through osseo-fibrous tunnels formed by the annular and cruciform pulleys and covered by the flexor retinaculum. In a "trigger-finger", mechanical overuse causes thickening of the annular pulley, narrowing of the osseofibrous tunnel, and stenosing tenosynovitis of the adjacent flexor tendons ${ }^{(28)}$ (Fig. 18).

\section{Pulley injuries}

Most injuries of the annular pulleys (for anatomy, see Fig. 19A) happen in rock climbers. The A2 pulley of the third and fourth digits is most commonly affected ${ }^{(27)}$. Typically, excessive stress with flexor tendon contraction against the pulley system causes pulley injury. A common risk factor for pulley injuries is the "crimp position", with $90^{\circ}$ or more flexion of the PIP joint and slight DIP joint hyperextension. Using high-resolution US probes, a normal annular pulley is identified as a very thin $(0.3-0.5 \mathrm{~mm})$ band surrounding the flexor tendons on their volar side (Fig. 19B) ${ }^{(5)}$. Pulley ligaments are best visualized on transverse scanning planes: the normal US 


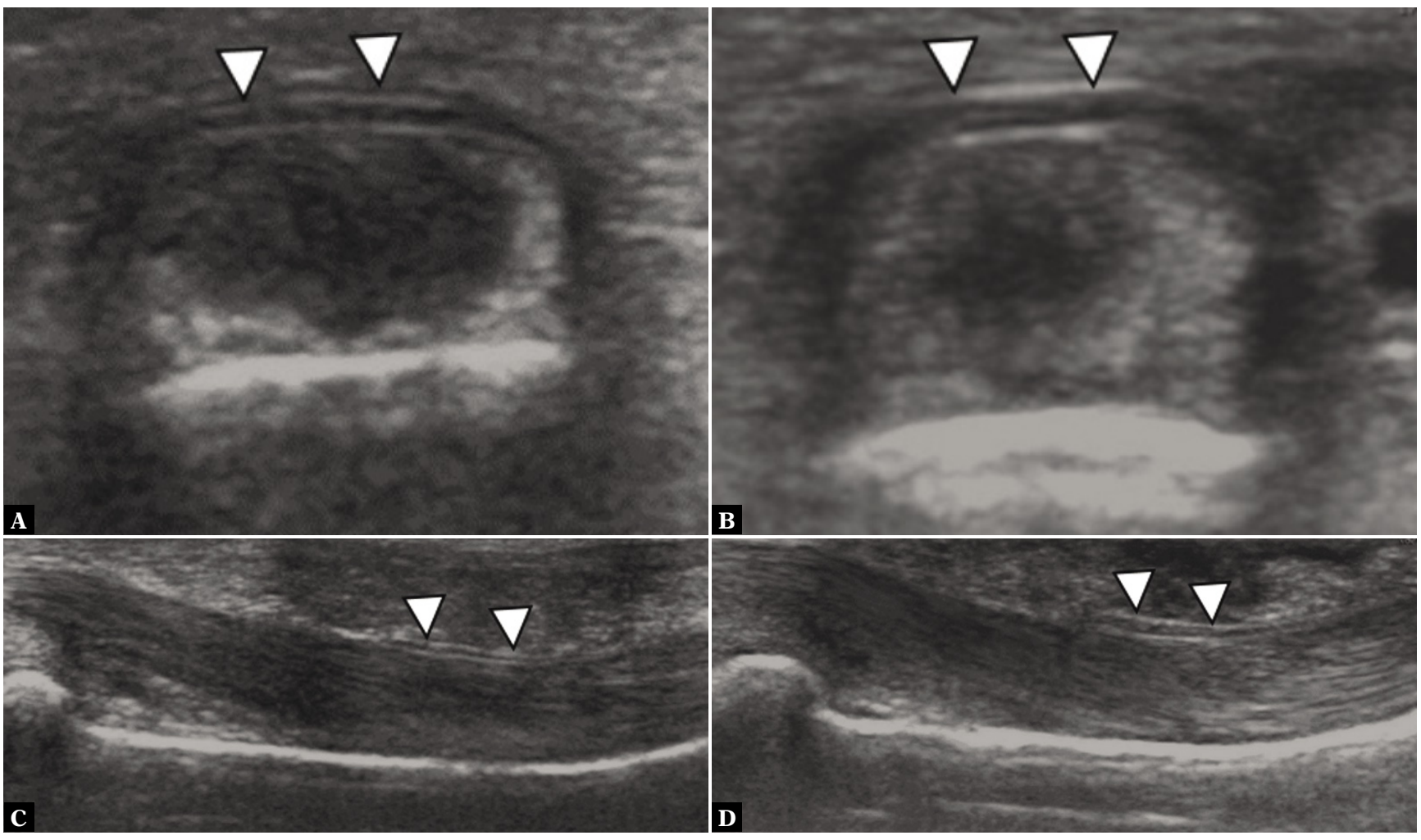

Fig. 20. Transverse (A) and sagittal (C) US with normal thin appearance of A2-pulley (white arrowheads) with normal tendon-to-bone distance (TBD) in a non-climber. Transverse (B) and sagittal (D) US shows thickened A2 ligament (white arrowheads) due to chronic overuse in a climber. Images courtesy of Prof. A. Schweizer, Handsurgery, Balgrist University Hospital

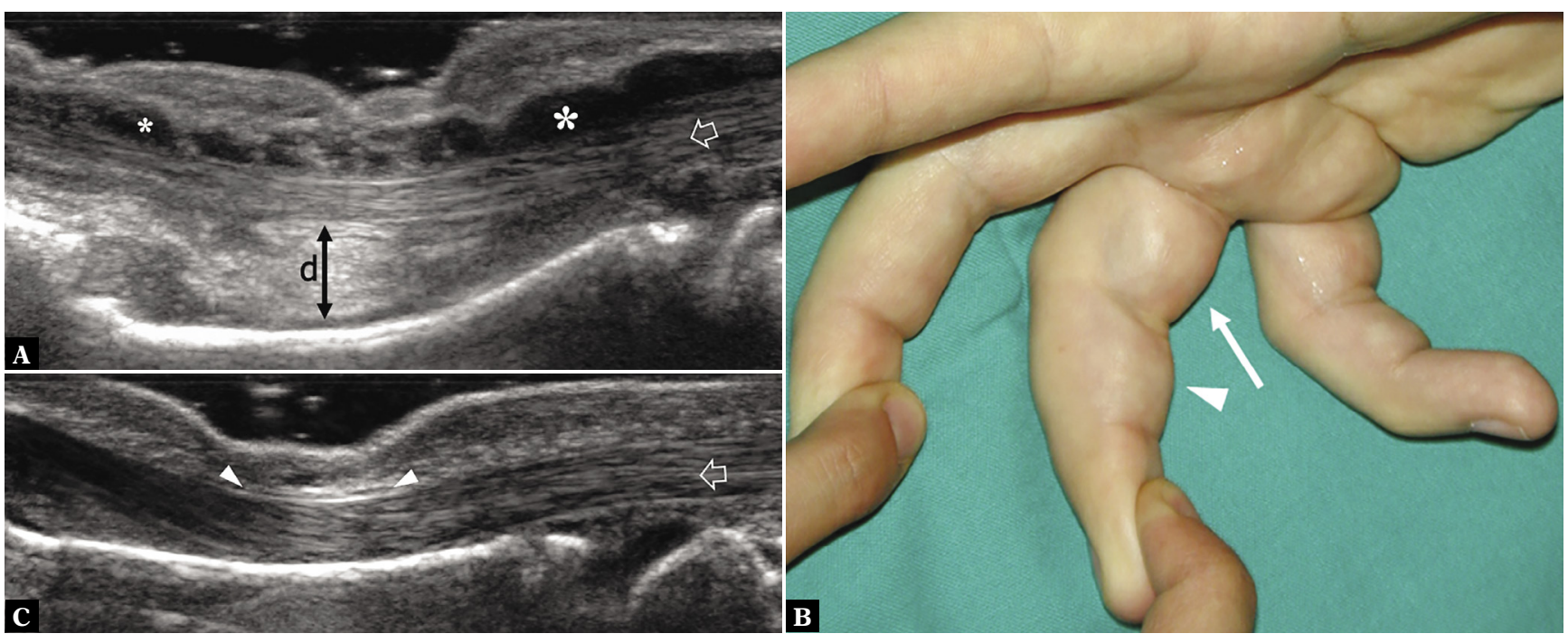

Fig. 21. Annular pulley injury. A. Longitudinal 17-5 MHz US image obtained over the injured right long finger of a 15-year-old boy during resisted flexion of the distal interphalangeal joint demonstrates bowstringing and volar displacement of the flexor tendons (arrows) secondary to acute rupture of the A2-pulley. In this particular case, there was also a combined injury of the A3- and A4-pulleys (not shown). An increased tendon-to-bone distance (>2 mm, double arrow), is seen over the proximal phalanx. Note an abundant effusion (asterisks) distending the tenosynovial sheath. B. Corresponding photograph shows volar soft-tissue swelling over the proximal (thin arrow) and middle (arrowhead) phalanges due to bowstringing of the flexor tendons. C. Longitudinal 17-5 MHz. US image over the left long finger illustrates the normal A2-pulley as a thin hypoechoic band (white arrowheads) retaining the flexor tendons (arrow) against the shaft of the proximal phalanx

appearance of an annular pulley is hyperechoic on the volar side and hypoechoic on the lateral side (the latter due to anisotropy). The thickness of the annular pulleys is often increased (1.5-fold) in asymptomatic climbers, when compared to non-climbers ${ }^{(32)}$ (Fig. 20). This structural adaptation simplifies US visualization of the pulley 
bands. Annular pulley injuries include pulley strains and partial or complete ruptures, involve one or multiple pulleys, and often result in variable bowstringing of the flexor tendons. Clinically, bowstringing is typically only visible when multiple injuries have occured in the A2, A3, and A4 pulleys ${ }^{(33)}$. With imaging (US and MRI) the detection of minor degrees of bowstringing is more sensitive ${ }^{(34)}$. The tendon-to-bone distance (TBD) increases in cases with bowstringing. US with forced flexion is used to determine the site of maximal volar bowstringing (MVB) and to diagnose which pulley has ruptured. In an A2 pulley rupture, MVB occurs over the proximal phalanx. In an A3 pulley rupture, MVB occurs at the distal proximal phalanx at level of the volar plate. In an A4 pulley rupture, MVB occurs over the middle part of the middle phalanx. US should be performed along the finger's long axis to detect tendon bowstringing and measure TBD (Fig. 21). The transverse scanning plane is best for assessment of the site of pulley detachment and visualization of any small osseous avulsion fragments.

Dynamic US is used to evaluate any instability of the flexor system associated with pulley injuries. Volar tendon displacement relative to the bone is measured at rest and in forced flexion. The normal TBD at the level of the A2 pulley ligament is $<2 \mathrm{~mm}^{(31)}$ (Fig. 19). In a complete, isolated A2 pulley tear, the TBD at the level of the

\section{References}

1. de Jong JP, Nguyen JT, Sonnema AJ, Nguyen EC, Amadio PC, Moran SL: The incidence of acute traumatic tendon injuries in the hand and wrist: a 10-year population-based study. Clin Orthop Surg 2014; 6: 196-202.

2. Schoffl V, Heid A, Kupper T: Tendon injuries of the hand. World J Orthop 2012; 3: 62-69.

3. De Maeseneer M, Meng J, Marcelis S, Jager T, Provyn S, Shahabpour M: Ultrasound anatomy of the fingers: flexor and extensor system with emphasis on variations and anatomical detail. J Ultrason 2020; 20: e122-e128.

4. Kleinert HE, Verdan C: Report of the Committee on Tendon Injuries (International Federation of Societies for Surgery of the Hand). J Hand Surg Am 1983; 8: 794-798.

5. Rosskopf AB, Taljanovic MS, Sconfienza LM, Gitto S, Martinoli C, Picasso R et al.: Pulley, Flexor, and Extensor Tendon Injuries of the Hand. Semin Musculoskelet Radiol 2021; 25: 203-215.

6. Bianchi S, Martinoli C, Abdelwahab IF: Ultrasound of tendon tears. Part 1: general considerations and upper extremity. Skeletal Radiol 2005; 34: 500-512.

7. Gitto S, Draghi AG, Draghi F: Sonography of non-neoplastic disorders of the hand and wrist tendons. J Ultrasound Med 2018; 37: 51-68.

8. Alla SR, Deal ND, Dempsey IJ: Current concepts: mallet finger. Hand (N Y) 2014; 9: 138-144.

9. Schweitzer TP, Rayan GM: The terminal tendon of the digital extensor mechanism: Part II, kinematic study. J Hand Surg Am 2004; 29: 903-908.

10. Lee SA, Kim BH, Kim S-J, Kim JN, Park S-Y, Choi K: Current status of ultrasonography of the finger. Ultrasonography 2016; 35: 110-123.

11. Vassa R, Garg A, Omar IM: Magnetic resonance imaging of the wrist and hand. Pol J Radiol 2020; 85: e461-e488.

12. Westerheide E, Failla JM, van Holsbeeck M, Ceulemans R: Ultrasound visualization of central slip injuries of the finger extensor mechanism. J Hand Surg Am 2003; 28: 1009-1013.

13. Willekens I, Kichouh M, Boulet C, De Maeseneer M, Clarys JP, de Mey $\mathrm{J}$ : Ultrasound follow-up of posttraumatic injuries of the sagittal band proximal phalanx is $>2 \mathrm{~mm}$ at rest and $>4 \mathrm{~mm}$ during forced flexion ${ }^{(31)}$. In combined A2, A3, and A4 pulley tears, the TBD is $>2 \mathrm{~mm}$ at rest and $>5.5 \mathrm{~mm}$ during forced flexion (Fig. 21). US during forced flexion is particularly helpful for differentiation of isolated A2 pulley tears and combined A2 and A3 pulley tears ${ }^{(5,34)}$. Diagnosis of partial pulley ruptures is more challenging because they produce little to no tendon displacement. In such cases, the affected pulley is hypoechoic and swollen. Abnormal vascularization can be detected in both the tendons and pulleys in acute conditions using Doppler examination. A classification system adapted from Schöffl and Schöffl (grade I-IV) for grade I to IV flexor pulley injuries is used to guide treatment decision making ${ }^{(35)}$. While grade I to III injuries (i.e., strain, partial rupture, and complete rupture of a single pulley) can be managed conservatively without operation, Grade IV injuries (i.e., multiple pulley ruptures or a single rupture of A2 or A3 with concomitant trauma (ligaments/lumbrical muscles)) require surgery.

\section{Conflict of interest}

Authors do not report any financial or personal connections with other persons or organizations, which might negatively affect the contents of this publication and/or claim authorship rights to this publication.

of the dorsal hood treated by a conservative approach. Eur J Radiol 2015; 84: 278-283.

14. Rayan GM, Murray D: Classification and treatment of closed sagittal band injuries. J Hand Surg Am 1994; 19: 590-594.

15. Jacob D, Cohen M, Bianchi S: Ultrasound imaging of non-traumatic lesions of wrist and hand tendons. Eur Radiol 2007; 17: 2237-2247.

16. Plotkin B, Sampath SC, Sampath SC, Motamedi K: MR Imaging and US of the wrist tendons. Radiographics 2016; 36: 1688-1700.

17. Kamel M, Moghazy K, Eid H, Mansour R: Ultrasonographic diagnosis of de Quervain's tenosynovitis. Ann Rheum Dis 2002; 61: 1034-1035.

18. Goel R, Abzug JM: de Quervain's tenosynovitis: a review of the rehabilitative options. Hand (N Y) 2015; 10: 1-5.

19. Montechiarello S, Miozzi F, D'Ambrosio I, Giovagnorio F: The intersection syndrome: ultrasound findings and their diagnostic value. J Ultrasound 2010; 13: 70-73.

20. Draghi F, Bortolotto C: Intersection syndrome: ultrasound imaging. Skeletal Radiol 2014; 43: 283-287.

21. Draghi F, Bortolotto C, Draghi AG, Gregoli B: Musculoskeletal sonography for evaluation of anatomic variations of extensor tendon synovial sheaths in the wrist. J Ultrasound Med 2015; 34: 1445-1452.

22. Ghazal L, Nabi M, Little C, Teh J: Ultrasound assessment of extensor pollicis longus tendon rupture following distal radius fracture: a sonographic and surgical correlation. J Ultrason 2020; 20: E1-E5.

23. Taljanovic MS, Melville DM, Gimber LH, Scalcione LR, Miller MD, Kwoh CK et al.: High-resolution US of rheumatologic diseases. Radiographics 2015; 35: 2026-2048.

24. Kim CH: Anomalous extensor indicis proprius muscle. Arch Plast Surg 2013; 40: 79-81.

25. Yoo MJ, Chung KT, Kim JP, Kim MJ, Lee KJ: Tendon impingement of the extensor digiti minimi: clinical cases series and cadaveric study. Clin Anat 2012; 25: 755-761.

26. Tanaka T, Kamada H, Ochiai N: Extensor tendon rupture in ring and little fingers with DRUJ osteoarthritis without perforating the DRUJ capsule. J Orthop Sci 2006; 11: 221-223. 
27. Shapiro LM, Kamal RN: Evaluation and treatment of flexor tendon and pulley injuries in athletes. Clin Sports Med 2020; 39: 279-297.

28. Bianchi S, Martinoli C, de Gautard R, Gaignot C: Ultrasound of the digital flexor system: Normal and pathological findings(). J Ultrasound 2007; 10: 85-92.

29. Klauser A, Stadlbauer KH, Frauscher F, Herold M, Klima G, Schirmer M et al.: Value of transducer positions in the measurement of finger flexor tendon thickness by sonography. J Ultrasound Med 2004; 23: 331-337.

30. Ruchelsman DE, Christoforou D, Wasserman B, Lee SK, Rettig ME: Avulsion injuries of the flexor digitorum profundus tendon. J Am Acad Orthop Surg 2011; 19: 152-162.

31. Klauser A, Frauscher F, Bodner G, Cihak C, Gabl M, Schocke M et al.: [Value of high-resolution ultrasound in the evaluation of finger injuries in extreme sport climbers]. Ultraschall Med 2000; 21: 73-78.
32. Jones G, Johnson MI: A critical review of the incidence and risk factors for finger injuries in rock climbing. Curr Sports Med Rep 2016; 15: 400-409.

33. Klauser A, Bodner G, Frauscher F, Gabl M, Zur Nedden D: Finger injuries in extreme rock climbers. Assessment of high-resolution ultrasonography. Am J Sports Med 1999; 27: 733-737.

34. Klauser A, Frauscher F, Bodner G, Halpern EJ, Schocke MF, Springer P et al.: Finger pulley injuries in extreme rock climbers: depiction with dynamic US. Radiology 2002; 222: 755-761.

35. Schoffl VR, Schoffl I: Injuries to the finger flexor pulley system in rock climbers: current concepts. J Hand Surg Am 2006; 31: 647-654. 